\title{
Control par calculado difuso basado en pasividad para seguimiento de trayectorias de robots manipuladores
}

\author{
S.M. Orozco-Soto ${ }^{1}$, J.C. Ramos-Fernández ${ }^{2}$ \\ ${ }^{1}$ Universidad Tecnológica del Norte de Aguascalientes (UTNA), \\ Aguascalientes, México \\ ${ }^{2}$ Universidad Politécnica de Pachuca (UPP), \\ Hidalgo, México \\ jramos@upp.edu.mx, santos.orozco@utna.edu.mx
}

\begin{abstract}
Resumen. En este trabajo se presenta una alternativa para mejorar la técnica de control Par-Calculado para seguimiento de trayectorias de robots manipuladores. La estrategia de control propuesta utiliza lógica difusa para calcular la compensación requerida por el Control Par Calculado clásico ante variaciones en los parámetros del robot tales como masa o fricción. La innovación de esta propuesta es que la mejora del controlador Par Calculado se realiza en la parte de la compensación de las dinámicas en lugar de la parte tipo PD de la estructura del controlador. La estabilidad asintótica global se garantiza utilizando la teoría de la pasividad y el método directo de Lyapunov en el diseño del controlador. Los experimentos de simulación se realizaron utilizando el modelo dinámico de un robot manipulador de 2 GDL en configuración planar con presencia de pares gravitacionales. Los resultados satisfactorios, así como la sencillez de la estructura del controlador motivan a la implementación de esta propuesta en robots reales utilizando sistemas embebidos.
\end{abstract}

Palabras clave: par calculado, lógica difusa, seguimiento de trayectoria, pasividad, método directo de Lyapunov.

\section{Introducción}

El controlador Par Calculado es preferido para seguimiento de trayectorias de robots manipuladores debido a que garantiza la estabilidad asintótica global y calcula la cantidad de energía exacta que requieren los actuadores de las articulaciones de los robots para llevar a cabo las trayectorias deseadas [1-3]. Sin embargo, este controlador es susceptible a variaciones en el modelo del robot, incertidumbre en los parámetros del mismo o dinámicas no modeladas, por lo que se han desarrollado diversas alternativas para mejorar el desempeño de esta técnica, tales como complementos robustos[4], algoritmos genéticos [5] o estrategias basadas en lógica difusa [6-9]. La mayoría de estas mejoras consisten en la adaptación de la parte proporcional-derivativa (PD) de la estructura del controlador en lugar de adaptar la parte de la compensación de las dinámicas del robot. En este trabajo, se presenta una alternativa para mejorar el 
rendimiento del control Par Calculado ante variaciones en los parámetros del robot utilizando lógica difusa. La estrategia de control propuesta fue diseñada utilizando las propiedades generales del modelo dinámico de robots manipuladores, la Teoría de Pasividad y el Segundo Método de Lyapunov, para garantizar estabilidad asintótica global durante el seguimiento de trayectoria. Para probar el controlador diseñado, se llevaron a cabo algunos experimentos en simulación utilizando el modelo dinámico de un robot manipulador de 2 grados de libertad (GDL) en configuración planar afectado por los pares gravitacionales. Los resultados satisfactorios del controlador propuesto motivan a la implementación de esta alternativa utilizando un microcontrolador o un sistema embebido para controlar un robot real. Este artículo está organizado de la siguiente manera: en la Sección 2, se presentan algunas propiedades del modelo dinámico de robots manipuladores, que son útiles para el diseño del controlador. En la Sección 3, se plantea el problema de la susceptibilidad del Control Par Calculado a las variaciones en los parámetros; asimismo, se formulan los objetivos de control. La Sección 4 describe el diseño del controlador propuesto utilizando lógica difusa y la Teoría de Lyapunov. Los resultados satisfactorios a nivel simulación se presentan en la Sección 5. Finalmente, en la Sección 6 se presentan las conclusiones.

\section{Propiedades del modelo dinámico de robots manipuladores}

En esta sección, se presentan algunas propiedades del modelo dinámico de robots manipuladores que son útiles para el diseño del controlador. La dinámica de los robots manipuladores puede ser representada matemáticamente por [10]:

$$
D(q) \ddot{q}+C(q, \dot{q}) \dot{q}+G(q)+F(\dot{q})=\tau,
$$

donde $D(q) \in \mathbb{R}^{n \times n}$ es la matriz de fuerzas inerciales, $C(q, \dot{q}) \in \mathbb{R}^{n \times n}$ es la matriz de fuerzas centrípetas y de Coriolis, $G(q) \in \mathbb{R}^{n}$ es el vector de pares gravitacionales, $F(\dot{q}) \in \mathbb{R}^{n}$ es el vector de fricción y $\tau \in \mathbb{R}^{n}$ es el vector de entradas de control. El modelo dinámico (1) cuenta con las siguientes propiedades:

Propiedad 1. La matriz de fuerzas inerciales es simétrica y definida positiva, i.e. [11]:

$$
\begin{gathered}
D(q) \equiv D(q)^{T}, \\
D(q)>0 .
\end{gathered}
$$

Propiedad 2. La matriz de fuerzas inerciales y la matriz de fuerzas centrípetas y de Coriolis tienen la siguiente relación (propiedad de antisimetría) [12]:

$$
\dot{\mathrm{q}}^{\mathrm{T}}\left[\frac{1}{2} \dot{\mathrm{D}}(\mathrm{q})-\mathrm{C}(\mathrm{q}, \dot{\mathrm{q}})\right] \dot{\mathrm{q}}=0,
$$

Propiedad 3. El modelo dinámico (1) puede ser parametrizado como [12]:

$$
D(q) \ddot{q}+C(q, \dot{q}) \dot{q}+G(q)+F(\dot{q})=\mathrm{Y}(\mathrm{q}, \dot{\mathrm{q}}, \ddot{\mathrm{q}})^{\mathrm{T}} \Phi
$$


donde $\mathrm{Y}(\mathrm{q}, \dot{\mathrm{q}}, \ddot{\mathrm{q}})^{\mathrm{T}} \in \mathbb{R}^{\mathrm{n} \times \mathrm{p}}$ es una matriz de regresión y $\Phi \in \mathbb{R}^{\mathrm{n}}$ es un vector conformado por parámetros constantes.

Propiedad 4. La energía total del sistema robótico (1) está dada por su ecuación Hamiltoniana como se muestra a continuación [12]:

$$
H(q, \dot{q})=\frac{1}{2} \dot{q}^{T} D(q) \dot{q}+G(q),
$$

Derivando (5) se obtiene:

$$
\dot{\mathrm{H}}(\mathrm{q}, \dot{\mathrm{q}})=\dot{\mathrm{q}}^{\mathrm{T}} \tau,
$$

Al integrar ambos lados de (6), se puede observar que el sistema (1) es pasivo, es decir:

$$
\mathrm{H}(\mathrm{q}, \dot{\mathrm{q}})-\mathrm{H}(0)=\int_{0}^{\mathrm{t}} \dot{\mathrm{q}}^{\mathrm{T}} \tau \mathrm{dt} .
$$

\section{Formulación del problema}

El Control Par Calculado es preferido para seguimiento de trayectoria de robots manipuladores debido a que calcula la energía exacta para llevar a cabo la tarea deseada [3]; este controlador se representa matemáticamente como [11]:

$$
\tau=K_{P} \tilde{q}+K_{D} \dot{\tilde{q}}+D(q) \ddot{q}_{d}+C(q, \dot{q}) \dot{q}_{d}+G(q)+F(\dot{q}),
$$

donde $\dot{q}_{d} \in \mathbb{R}^{n}$ y $\ddot{q}_{d} \in \mathbb{R}^{n}$ son los vectores de velocidades y aceleraciones deseadas respectivamente. Aplicando la Propiedad 3, el controlador (8) se puede expresar como sigue [12]:

$$
\tau=K_{P} \tilde{q}+K_{D} \dot{\tilde{q}}+\mathrm{Y}\left(\mathrm{q}, \dot{q}_{d}, \ddot{q}_{d}\right)^{\mathrm{T}} \Phi .
$$

Nótese que este controlador depende directamente del modelado adecuado y de la correcta identificación paramétrica del robot. Sin embargo, si alguno de los parámetros del vector $\Phi$, tales como la masa o la fricción, varía, el controlador no alcanzará la posición deseada o no seguirá la trayectoria requerida debido a que fue diseñado para calcular el par necesario de acuerdo a diferentes valores de los parámetros del robot. De esta manera, se establecen los siguientes objetivos de control:

Objetivo de control 1: Asegurar que el error de posición $\tilde{\mathrm{q}} \rightarrow 0$ mientras $t \rightarrow \infty$. El error de posición está dado por: $\tilde{\mathrm{q}}=\mathrm{q}_{\mathrm{d}}-\mathrm{q}$.

Objetivo de control 2: Si alguno de los parámetros del vector $\Phi$ cambia, adaptar el controlador (9) para garantizar que el Objetivo de control 1 se cumpla. 
El Objetivo de control puede alcanzarse utilizando del Control Par Calculado clásico (9), ya que garantiza la estabilidad global asintótica durante el seguimiento de trayectoria. Para lograr el Objetivo de control 2, se propone utilizar una versión adaptable del controlador (9), la cual está basada en lógica difusa para compensar variaciones en los parámetros del modelo del robot manipulador.

\section{Diseño del control par calculado difuso}

En esta sección, se presenta el diseño de la técnica de control propuesta para alcanzar los objetivos de control mencionados en la sección anterior. Dicha propuesta es una versión adaptable del Control Par Calculado (9), misma que utiliza lógica difusa para especificar el grado de compensación que requiere el controlador ante una variación en los parámetros del modelo del robot. La variación en los parámetros es detectada utilizando un índice de variación, el cual es también la variable de entrada del sistema difuso; la salida del sistema difuso es una ganancia variable agregada al Control Par Calculado, la cual es útil para aumentar o disminuir energía al sistema dependiendo de las variaciones en los parámetros del robot y del par requerido para desarrollar la tarea deseada.

\subsection{Variable fusificada}

La variable utilizada como entrada del sistema difuso es el índice de variación dado por:

$$
\Delta \Phi=\frac{\left\|\Phi_{R}\right\|}{\|\Phi\|}
$$

donde $\left\|\Phi_{R}\right\|$ es la norma Euclidiana del vector de parámetros con variaciones y $\|\Phi\|$ es la norma Euclidiana del vector de parámetros original. En la práctica, las normas de los vectores pueden ser sustituidas por mediciones de par. De esta forma, el índice de variación $\Delta \Phi$ se introduce al sistema difuso para calcular la energía requerida por los actuadores para realizar la tarea deseada en caso de una variación en los parámetros del robot.

\subsection{Particiones difusas}

El universo de discurso del sistema difuso fue seleccionado de acuerdo a los posibles valores del índice de variación, desde 0.6 , que significa casi la mitad del par requerido por los actuadores, hasta 2, lo cual supone la posibilidad de duplicar dicho par. Se decidió utilizar 5 particiones difusas para fusificar el índice de variación, mismas que se ilustran en la Figura 1. La partición High down sugiere una pérdida considerable de masa o de fricción en el robot, mientras que High up supone un incremento significativo en la masa o la fricción. Low up y Low down abordan cambios razonables de los parámetros incluyendo rangos de hasta $-20 \%$ y hasta $+30 \%$ del par requerido. La partición Minimum maneja variaciones de hasta \pm 5 . Nótese que todas las particiones 
están configuradas de la forma Hard Fuzzy, lo cual significa que la suma de todos los valores de pertenencia da por resultado 1; dicha configuración facilita la implementación de este controlador en la mayoría de los dispositivos programables.

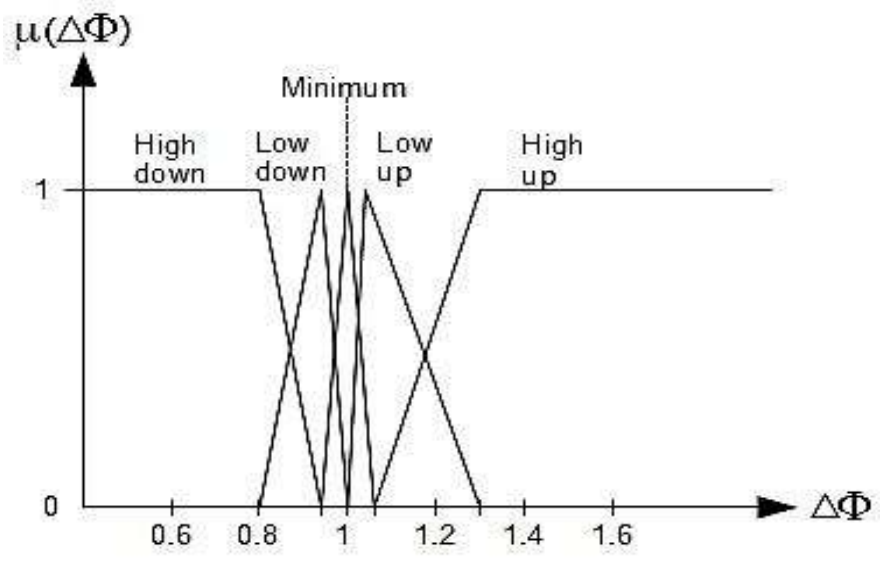

Fig. 1. Particiones difusas y universo de discurso del índice de variación.

\subsection{Reglas y superficie difusas}

Las reglas difusas fueron creadas realizando un mapeo del valor de pertenencia para cada partición difusa del índice de variación, hacia un valor duro, el cual es una ganancia llamada $k_{f}$. Las reglas difusas realizadas se pueden observar en la Tabla 1. Obsérvese que para pérdidas de masa o de fricción, el par debe ser reducido. Para incrementos de dichos parámetros, la compensación también debe incrementar. Para el caso de variaciones mínimas, el controlador no se ve afectado considerablemente, por lo que la parte tipo PD de la estructura del mismo debe ser capaz de manejar dichas perturbaciones; de esta forma, al no existir compensación, $k_{f}=1$.

Tabla 1. Reglas difusas.

\begin{tabular}{|l|}
\hline Si $\Delta \Phi$ es HIGH DOWN, entonces, $k_{f}$ es 0.2 \\
\hline Si $\Delta \Phi$ es LOW DOWN, entonces, $k_{f}$ es 0.8 \\
\hline Si $\Delta \Phi$ es MINIMUM, entonces, $k_{f}$ es 1 \\
\hline Si $\Delta \Phi$ es LOW UP, entonces, $k_{f}$ es 1.2 \\
\hline Si $\Delta \Phi$ es HIGH UP, entonces, $k_{f}$ es 2 \\
\hline
\end{tabular}

La superficie difusa que representa gráficamente el mapeo del índice de variación hacia la ganancia de compensación por medio de las reglas difusas se ilustra en la Figura 2 , donde se puede apreciar que el máximo valor de $\boldsymbol{k}_{\boldsymbol{f}}$ es capaz de compensar la energía necesaria incluso si el par incrementa al doble. En la práctica, el éxito del controlador 
depende directamente de la capacidad de los actuadores y de la electrónica de potencia para suministrar la energía calculada.

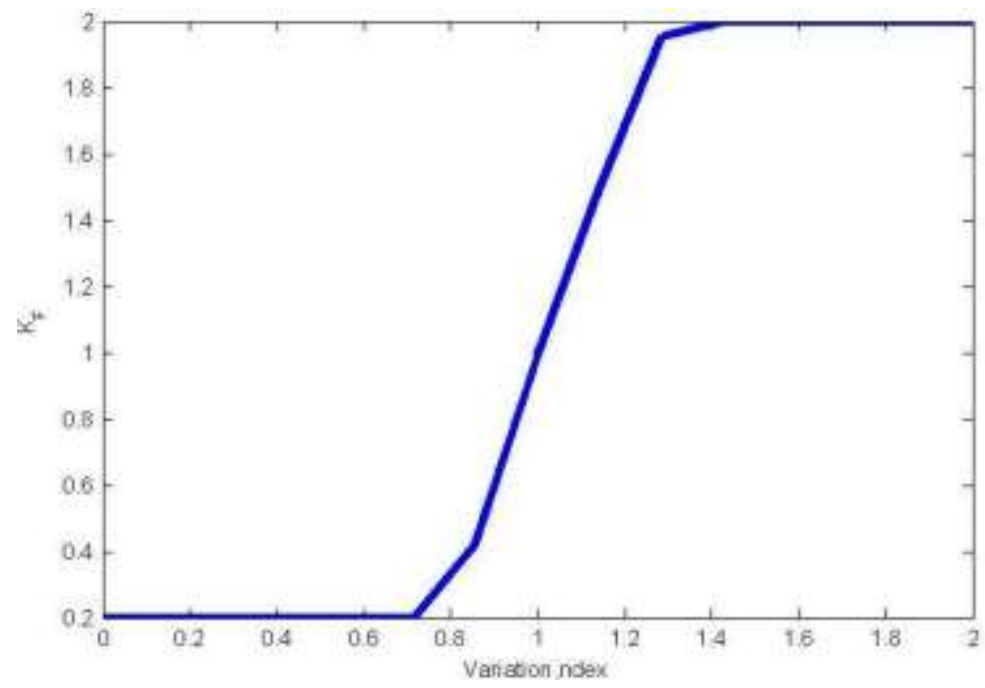

Fig. 2. Superficie difusa.

\subsection{Defusificación}

El método de defusificación seleccionado es la media ponderada, misma que se representa de la siguiente forma [13-14]:

$$
K_{F}=\frac{\sum_{i=1}^{5} \mu_{i}(\Delta \Phi) k_{f_{i}}}{\sum_{i=1}^{5} \mu_{i}(\Delta \Phi)},
$$

donde $K_{F}$ es una salida escalar que se utiliza como ganancia en el controlador, $i$ es el número de particiones difusas, $\mu_{i}(\Delta \Phi)$ es el valor de pertenencia del índice de variación (10) para cada partición difusa y $k_{f_{i}}$ es la salida correspondiente para cada partición. La ganancia $K_{F}$ es el valor duro que representa la compensación requerida para mejorar el rendimiento del Control Par Calculado (9) ante variaciones en los parámetros del robot manipulador.

\subsection{Diseño del controlador}

El controlador fue diseñado utilizando el Método Directo de Lyapunov, mismo que se describe en el siguiente teorema [15]:

Teorema 4.1 (Estabilidad Global) Una función escalar $V$ del estado $\boldsymbol{x}$ debe tener derivadas continuas de primer orden tales que:

- $\quad V(\boldsymbol{x})$ sea definida positiva 
Control par calculado difuso basado en pasividad para seguimiento de trayectorias de robots ...

- $\quad \dot{V}(\boldsymbol{x})$ sea definida negativa

- $V(\boldsymbol{x}) \rightarrow 0$ mientras $\|\boldsymbol{x}\| \rightarrow \infty$.

De esta forma, se propone la siguiente función candidata a ser Lyapunov:

$$
V(q, \widetilde{q}, \dot{\tilde{q}})=\frac{1}{2} \dot{\tilde{q}}^{T} D(q) \dot{\tilde{q}}+\frac{1}{2} \tilde{q}^{T} K_{P} \tilde{q}
$$

Como $K_{P}$ es una matriz diagonal constante, la función (12) satisface la primer condición del Teorema 4.1. Derivando (12) se obtiene:

$$
\dot{V}(q, \dot{q}, \tilde{q})=\dot{\tilde{q}}^{T} D(q) \ddot{\tilde{q}}+\frac{1}{2} \dot{\tilde{q}}^{T} \dot{D}(q) \dot{\tilde{q}}+\dot{\tilde{q}}^{T} K_{P} \tilde{q}
$$

Cabe mencionar que $D(q) \ddot{\tilde{q}}=D(q) \ddot{q}_{d}-D(q) \ddot{q}$, por lo tanto:

$$
\begin{aligned}
& \dot{V}(q, \dot{q}, \tilde{q})=\dot{\tilde{q}}^{T} D(q) \ddot{q}_{d}-\dot{\tilde{q}}^{T}[\tau-C(q, \dot{q}) \dot{q}-G(q)-F(\dot{q})]+\frac{1}{2} \dot{\tilde{q}}^{T} \dot{D}(q) \dot{\tilde{q}} \\
& +\dot{\tilde{q}}^{T} K_{P} \tilde{q} .
\end{aligned}
$$

Como $C(q, \dot{q}) \dot{q}=C(q, \dot{q}) \dot{q}_{d}-C(q, \dot{q}) \dot{\tilde{q}}$, se puede sustituir en (14) como sigue:

$$
\begin{aligned}
& \dot{V}(q, \dot{q}, \tilde{q})=\dot{\tilde{q}}^{T} D(q) \ddot{q}_{d}-\dot{\tilde{q}}^{T}\left[\tau-C(q, \dot{q}) \dot{q}_{d}+C(q, \dot{q}) \dot{\tilde{q}}-G(q)-F(\dot{q})\right] \\
& +\frac{1}{2} \dot{\tilde{q}}^{T} \dot{D}(q) \dot{\tilde{q}}+\dot{\tilde{q}}^{T} K_{P} \tilde{q} .
\end{aligned}
$$

Aplicando la Propiedad de antisimetría:

$\dot{V}(q, \dot{q}, \tilde{q})=\dot{\tilde{q}}^{T}\left[-\tau+D(q) \ddot{q}_{d}+C(q, \dot{q}) \dot{q}_{d}+G(q)+F(\dot{q})+K_{P} \tilde{q}\right]$.

A pesar de que (19) no es una función definida negativa, puede ser forzada a serlo utilizando [12]:

$$
-\dot{\tilde{q}}^{T} K_{D} \tilde{q}=\dot{\tilde{q}}^{T}\left[-\tau+D(q) \ddot{q}_{d}+C(q, \dot{q}) \dot{q}_{d}+G(q)+F(\dot{q})+K_{P} \tilde{q}\right] .
$$

Entonces, la ley de control utilizada para satisfacer la segunda y tercera condición del Teorema 4.1 es:

$$
\tau=K_{P} \tilde{q}+K_{D} \dot{\tilde{q}}+K_{F} \mathrm{Y}\left(\mathrm{q}, \dot{q}_{d}, \ddot{q}_{d}\right)^{\mathrm{T}} \Phi
$$

Nótese que cuando $K_{F}=1$ (ausencia de compensación), el controlador (18) es el Control Par Calculado clásico (9).

\section{Resultados}

Los experimentos a nivel simulación se llevaron a cabo utilizando el modelo dinámico de un robot de 2 GDL afectado por pares gravitacionales con los siguientes parámetros: 
Tabla 2. Parámetros del robot manipulador.

\begin{tabular}{|c|c|c|}
\hline Parámetro & Símbolo & Valor \\
\hline Masa del eslabón 1 & $m_{1}$ & 2.8 \\
\hline Masa del eslabón 2 & $m_{2}$ & 1.75 \\
\hline Longitud del eslabón 1 & $l_{1}$ & 0.35 \\
\hline Longitud del eslabón 2 & $l_{2}$ & 0.2 \\
\hline $\begin{array}{c}\text { Longitud de la articulación 1 al centro de gravedad del } \\
\text { eslabón 1 }\end{array}$ & $l_{c 1}$ & 0.21 \\
\hline $\begin{array}{c}\text { Longitud de la articulación 2 al centro de gravedad del } \\
\text { eslabón 2 }\end{array}$ & $l_{c 2}$ & 0.14 \\
\hline Momento de inercia del eslabón 1 & $I_{1}$ & 0.45 \\
\hline Momento de inercia del eslabón 2 & $I_{2}$ & 0.33 \\
\hline Aceleración de la gravedad & $g$ & 9.81 \\
\hline Coeficiente de fricción de la articulación 1 & $f_{1}$ & 0.9 \\
\hline Coeficiente de fricción de la articulación 2 & $f_{2}$ & 0.9 \\
\hline
\end{tabular}

El vector $\Phi$ contiene todos los parámetros de la Tabla 2. La trayectoria que debe seguir el efector final del robot es un círculo con las siguientes ecuaciones paramétricas:

$$
\begin{aligned}
& \mathrm{x}_{\mathrm{d}}=0.3+0.15 \cos (\mathrm{t}) \\
& \mathrm{y}_{\mathrm{d}}=0.2+0.15 \cos (\mathrm{t})
\end{aligned}
$$

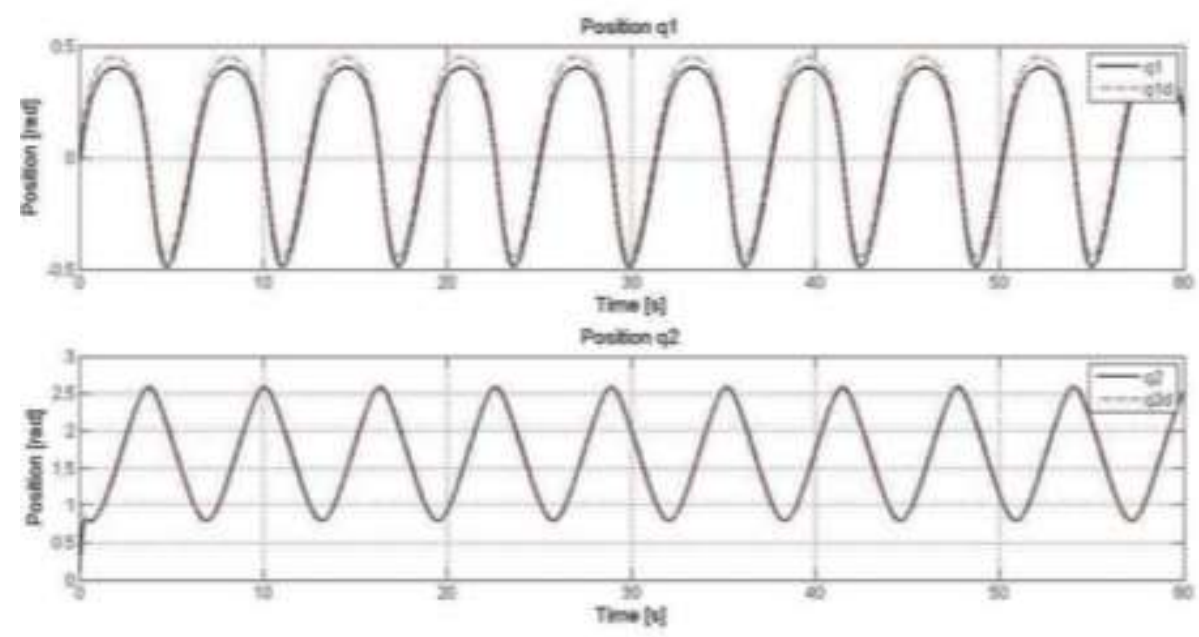

Fig. 3. Trayectorias articulares con Control Par Calculado clásico.

Lo cual sugiere que el robot debe realizar un círculo de $15 \mathrm{~cm}$ de radio. El tiempo de simulación es de 60 segundos. El primer experimento fue realizado variando la fricción de las articulaciones de 0.9 a 1.5 , utilizando el Control Par Calculado clásico; los resultados de este experimento se ilustran en la Figura 3 y en la Figura 4. 
En la Figura 3 se aprecia que las trayectorias articulares no corresponden a las referencia; asimismo, en la Figura 4 se observa que el robot no realiza el círculo debido a la falta de energía por parte de los actuadores.

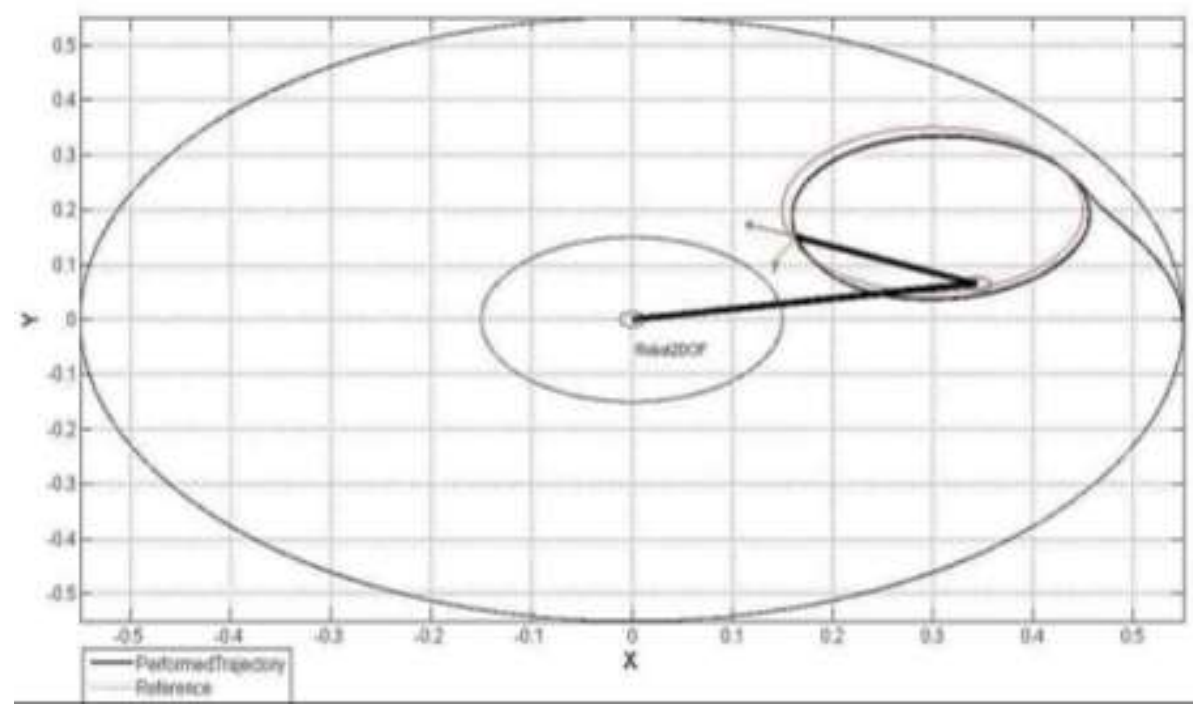

Fig. 4. Trayectoria del efector final con Control Par Calculado clásico.
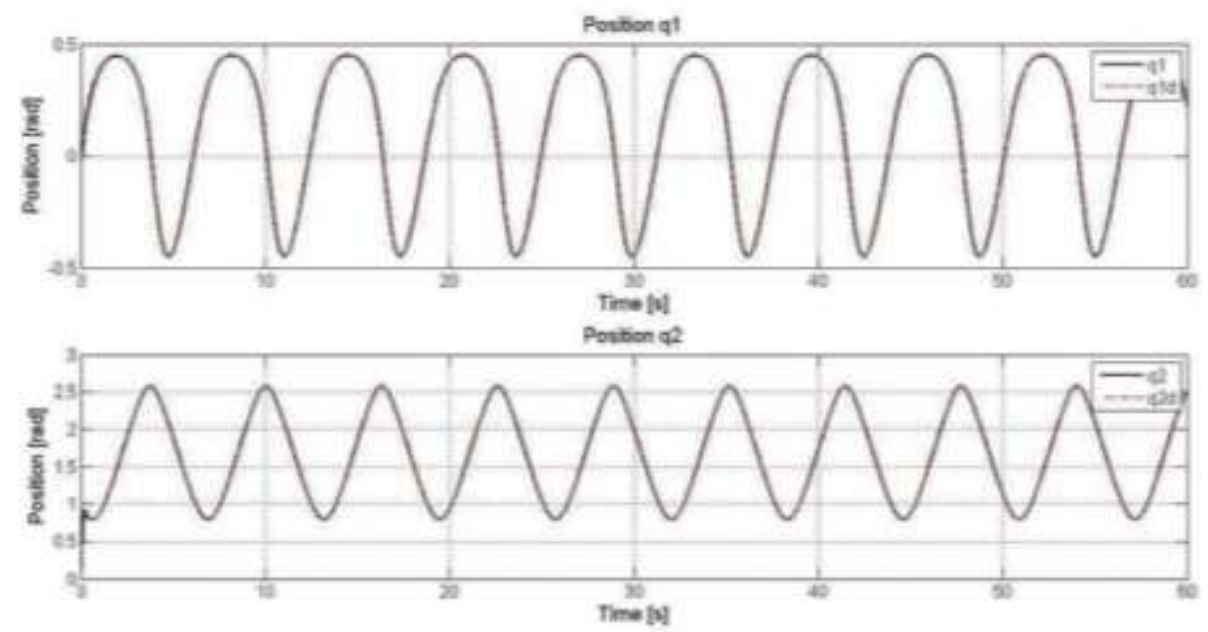

Fig. 5. Trayectorias articulares con Control Par Calculado difuso.

El segundo experimento se llevó a cabo utilizando el controlador propuesto en este trabajo, cuyos resultados se ilustran en la Figura 5 y en la Figura 6. En la Figura 5 se puede apreciar que las trayectorias articulares siguen correctamente las trayectorias 
deseadas. En la Figura 6 se observa como la trayectoria del efector final del robot corresponde a la trayectoria deseada.

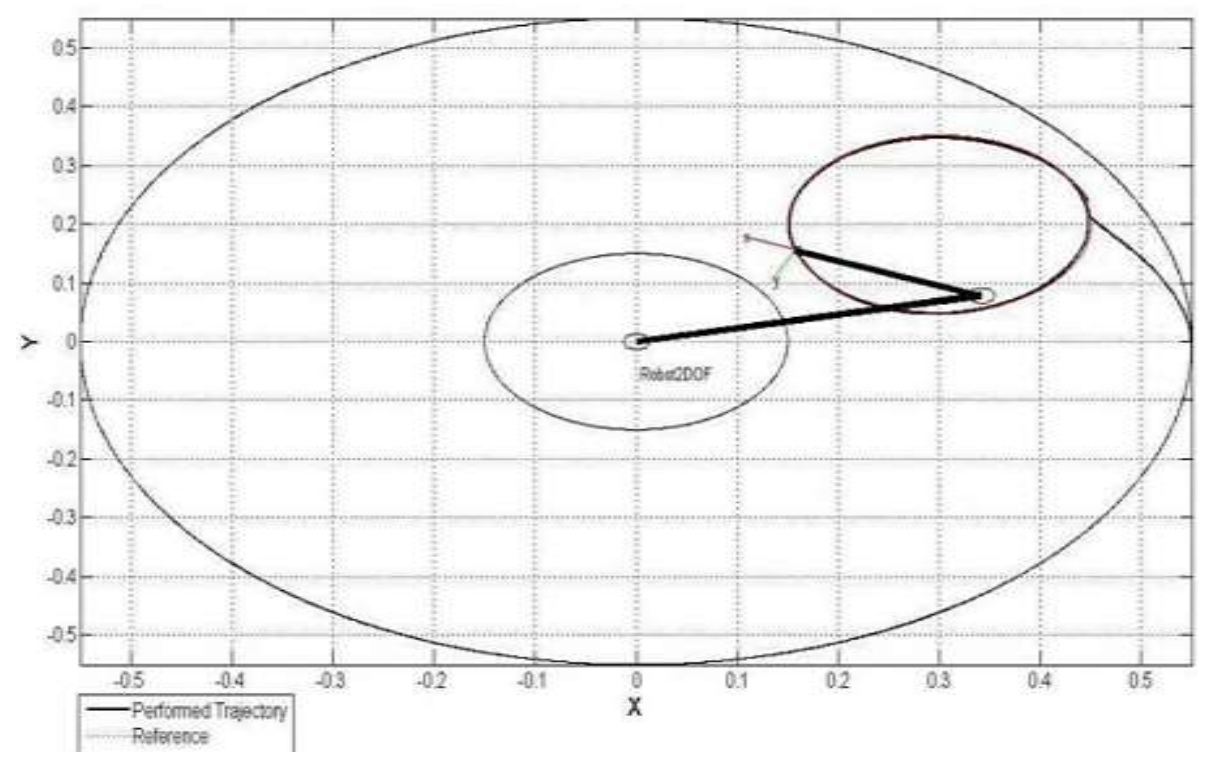

Fig. 6. Trayectoria del efector final con Control Par Calculado difuso.

\section{Conclusiones}

En este trabajo se presenta el diseño de una alternativa para mejorar el Control Par Calculado clásico utilizando lógica difusa. El controlador propuesto es capaz de compensar variaciones en los parámetros del robot tales como las masas de los eslabones o fricción en las articulaciones, utilizando la fusificación de un índice de variación, el cual se mapea hacia un dato duro que se utiliza como ganancia para la mejora del Control Par Calculado clásico. La estabilidad asintótica de este controlador se garantiza utilizando la Teoría de Pasividad y el Método Directo de Lyapunov para el diseño del mismo. La innovación que presenta esta estrategia de control es la mejora de la técnica clásica en la parte de la compensación de las dinámicas del robot en lugar realizarse en la parte tipo PD de la estructura.

Los resultados satisfactorios muestran que la alternativa propuesta es útil para compensar variaciones del robot cuando ya ha sido previamente parametrizado y puesto en marcha. En la práctica, el índice de variación puede ser calculado utilizando mediciones directas de par o de corriente en los actuadores. La sencillez de la estructura de este controlador y los resultados satisfactorios a nivel simulación motivan a su implementación utilizando microcontroladores comunes o hardware embebido; además, es recomendable asegurarse de que los actuadores y las interfaces de potencia utilizadas sean capaces de suministrar la energía calculada por el controlador propuesto para garantizar su correcto funcionamiento. 


\section{Referencias}

1. M.A. Llama, R. Kelly and V. Santibañez: Stable Computed-Torque Control of Robot Manipulators via Fuzzy Self-Tuning. IEEE Transactions on Systems, Man, and Cybernetics, Part B: Cybernetics, vol. 30, no. 1, pp. 143-150 (2000)

2. B. R. Markiewicz: Analysis of the Computed Torque Drive Method and Comparison with Conventional Position Servo for a Computer-Controlled Manipulator. California Institute of Technology, Jet Propulsion Laboratory, Tech. Rep. 33-601 (1973)

3. P.K. Khosla and T. Kanade: Real-Time Implementation and Evaluation of the Computed Torque Scheme. IEEE Robotics and Automation, vol. 5, no. 2, pp. 254-253 (1989)

4. H. Berghuis, H. Nijmeijer and P. Löhnberg: An Addendum on Robust Control of Robots by the Computed Torque Methods. Systems and Control Letters, vol. 18, pp. $403-407$ (1992)

5. B. Porter and N. N. Zadeh: Genetic Design of Computed-Torque/Fuzzy Logic Controllers for Robotic Manipulators. In: Proc. IEEE International Symposium on Intelligent Control (1995)

6. V. Santibañez, R. Kelly and M. Llama: Asymptotic Stable Tracking for Robot Manipulators via Sectorial Fuzzy Control. In: Proc. $15^{\text {th }}$ Triennial World Congress (2002)

7. S. Soltani and F. Piltan: Design Artificial Nonlinear Controller Based on Computed Torque Like Controller with Tunable Gain. World Applied Sciences Journal, vol. 14, no. 9, pp. 1306-1312 (2011)

8. F. Piltan, N. Sulaiman, A. Jalali and F. D. Narouei: Design of Model Free Adaptive Fuzzy Computed Torque Controller: Applied to Nonlinear Second Order System. International Journal of Robotics and Automation, vol. 2, no. 4, pp. 232-244 (2011)

9. Y. Chen, G. Ma, S. Lin and J. Gao: Adaptive Fuzzy Computed-Torque Control for Robot Manipulator with Uncertain Dynamics. International Journal of Advanced Robotic Systems, vol. 9 , no. 237, pp. 1-9 (2012)

10. M. W. Spong, S. Hutchinson and M. Vidyasagar: Robot Modeling and Control. John Wiley and Sons (2006)

11. R. Kelly y V. Santibañez: Control de Movimiento de Robots Manipuladores. Pearson Prentice Hall (2003)

12. J.P. Ordaz-Oliver, O.A. Domínguez-Ramírez, V. Parra-Vega y A. Jarillo-Silva: Diseño de Estrategias de Control Basadas en la Pasividad para Sistemas Euler-Lagrange Aplicado en Robots Manipuladores, Editorial Instituto Politécnico Nacional (2009)

13. S.M. Orozco-Soto: Intelligent Nonlinear PI Controller for a 3DOF Robot Manipulator Implemented on a DSP. In: Proc. of $2^{\text {nd }}$ International Congress on Instrumentation and Applied Sciences (ICIAS) (2012)

14. L.X. Wang: A Course on Fuzzy Systems and Control, Prentice Hall (1997)

15. J.E. Slotine and W. Li: Applied Nonlinear Control, Prentice Hall (1991) 